\title{
Closed management of displaced Type II odontoid fractures: more frequent respiratory compromise with posteriorly displaced fractures
}

\author{
Gregory J. Przybylski, M.D., James S. Harrop, M.D., AND \\ Alexander R. VACCARO, M.D. \\ Departments of Neurosurgery and Orthopedic Surgery, Jefferson Medical College, Thomas Jefferson \\ University Hospital, Philadelphia, Pennsylvania
}

\begin{abstract}
Object. Acute respiratory failure has been observed in patients after external immobilization for displaced odontoid fractures. The authors studied the frequency of respiratory deterioration in the acute management of displaced Type II odontoid fractures to identify patients at risk for respiratory failure.

Methods. The authors conducted a retrospective review of a consecutive series of 89 patients with odontoid fractures who were treated over a 5-year period to identify 53 patients with displaced Type II odontoid fractures. Patient demographics, degree of displacement, respiratory status, treatment method, and outcome were examined. Of the 32 patients with posteriorly displaced fractures, 13 experienced acute respiratory compromise, whereas only one of 21 patients with anteriorly displaced fractures had respiratory difficulties $(\mathrm{p}=0.0032)$. The average posterior displacement was $6.9 \mathrm{~mm}$. All 13 were initially managed using flexion traction for reduction of these fractures. Two of these patients died because of failure to emergently secure an airway during closed treatment of the fracture.

Conclusions. Frequent respiratory deterioration during acute closed reduction of posteriorly displaced Type II odontoid fractures was observed, whereas respiratory failure in patients with anteriorly displaced fractures was rare. The use of the flexed cervical position in the setting of retropharyngeal edema rather than the direction of the displacement may substantially increase the risk of respiratory failure. This may prompt early elective nasotracheal intubation during closed reduction of posteriorly displaced Type II odontoid fractures that require a flexed posture.
\end{abstract}

KEY WORDS • odontoid • axis - fracture - respiratory distress - traction

Odontoid fractures are frequently observed in patients who have experienced acute cervical spine trauma. However, despite significant displacement, these fractures are not commonly associated with spinal cord injury. Initial treatment typically includes skeletal traction for fracture reduction followed by external immobilization with or without surgical stabilization. Although the morbidity involved in this nonsurgical treatment is considered limited, rare complications of closed management have been reported. The purpose of this study was to determine the frequency of respiratory distress in patients with Type II odontoid fractures and to identify which factors increase the risk for respiratory compromise during acute management of this injury.

\section{CLINICAL MATERIAL AND METHODS}

A retrospective review of a consecutive series of patients admitted to the Delaware Valley Spinal Cord Injury Center at Thomas Jefferson University Hospital between January 1994 and July 1998 was performed. Eighty-nine of 166 patients with atlantoaxial fractures had odontoid fractures. Fifty-seven of 65 Type II fractures were displaced. Four patients who were intubated prior to admission were excluded: three with C-2 American Spinal Injury Association Grade A spinal cord injuries and one with polytrauma and hemodynamic instability; all had posteriorly displaced Type II fractures. The medical records and imaging studies of each patient were individually reviewed to determine patient age, mechanism of injury, fracture type and displacement, respiratory condition, treatment methods, and neurological status. Respiratory distress was defined as acute $\mathrm{SaO}_{2}$ of less than $80 \%$ after cervical manipulation, which resolved with the immediate cessation of traction manipulation or which required emergency intubation for airway management.

On admission, anteroposterior and lateral cervical, thoracic, and lumbar radiographs as well as an open mouth odontoid view were obtained on all patients. Axial computerized tomography scanning with sagittal and coronal reformatting was often performed to delineate further the type of upper cervical spine injury. Odontoid fracture displacement and angulation were measured from admission radiographs. Displacement was recorded as neg- 
ative for anterior and positive for posterior displacement. Fisher's exact and unpaired t-tests were used for statistical comparisons with the level of statistical significance set at $\mathrm{p}<0.05$.

\section{RESULTS}

There were 53 patients with displaced fractures (32 posteriorly and 21 anteriorly) among the 61 patients with Type II odontoid fractures. All patients were placed in acute skeletal traction to restore spinal alignment. Thirteen of the 32 patients with posteriorly displaced odontoid fractures experienced acute respiratory compromise during closed skeletal traction, whereas only one of 21 patients with anterior displacement experienced respiratory failure $(\mathrm{p}<0.0032)$. No patient with nondisplaced Type II odontoid fracture developed respiratory difficulty.

Of the 32 patients with posteriorly displaced odontoid fractures, 13 experienced respiratory distress during the acute phase $(<24$ hours) of closed reduction in flexion traction. Nine patients required immediate endotracheal intubation after acute $\mathrm{SaO}_{2}$. Initial endotracheal intubation was unsuccessful in four of these patients, prompting emergency tracheostomy. In two patients, an airway could not be secured initially and the patients sustained anoxic brain injury; medical care was subsequently withdrawn in both after discussion with their families. Only one patient with an anteriorly displaced fracture experienced respiratory distress; this was an 85-year-old woman with a 5-mm anteriorly displaced fracture who required acute intubation after desaturation during axial skeletal traction.

Demographics including age, sex, neurological condition, and associated spinal and nonspinal fractures were similar in patients with displaced fractures. Likewise, there was no difference in body habitus between the two groups. Most fractures were due to low velocity injuries such as a fall. Although no patient experienced neurological worsening due to cervical traction or manipulation, two patients suffered anoxic brain injury because of failure to secure an adequate airway and eventually had medical life support withdrawn. The death rate was higher in patients who experienced respiratory distress $(\mathrm{p}=$ 0.0038 ). The mean fracture displacement of the Type II posteriorly displaced odontoid fracture subtypes was 6.9 $\mathrm{mm}$ (range 1-18 $\mathrm{mm}$ ) in the patients with acute respiratory distress $(p<0.05)$ in comparison with the mean fracture displacement of the anteriorly displaced fractures of $4.2 \mathrm{~mm}$ (range -1 to $-8 \mathrm{~mm}$ ).

\section{DISCUSSION}

Odontoid fractures are common after cervical trauma, representing 10 to $15 \%$ of all cervical spine fractures. ${ }^{8}$ Because atlantoaxial instability was frequently observed to cause death in patients with untreated fractures, ${ }^{9}$ current treatment includes initial reduction with traction followed by halo vest immobilization and/or surgical stabilization. ${ }^{2,6}$ However, bone nonunion after halo vest immobilization has been reported in 4 to $63 \%$ of patients..$^{1-7,14}$ Factors associated with bone nonunion after rigid immobilization include patient age older than 40 years, displacements greater than $5 \mathrm{~mm}$, posteriorly displaced fractures, and patients with neurological deficits. ${ }^{6}$
Traction is often used to reduce displaced fractures. Although anteriorly displaced fractures typically require extension for reduction, posteriorly displaced fractures may require flexion for adequate reduction. An adjustable pulley on a traction frame can be used to deliver the appropriate combination of anterior and posterior vector forces based on the angulation and direction of the displaced fragment. Traction weight is usually initiated at 5 lbs after checking a radiograph to determine if excessive distraction from unrecognized ligamentous injury has occurred. ${ }^{13}$ Subsequently, traction weight is gradually increased until reduction of the displaced fragment is maintained without distraction. Lateral cervical radiographs are examined to access fracture alignment with each change in traction weight or vector direction. After reduction is achieved, the patient can be immobilized in an external orthosis such as a halo vest.

Most reported complications from traction are minor, including scalp infections, decubiti, screw loosening, and osteomyelitis. ${ }^{6}$ Typically, death and respiratory failure are not reported acute complications from "conservative therapy" with closed skeletal reduction. However, respiratory arrests resulting in death have been reported in numerous large series of patients treated with closed reduction. . $^{1,5-11}$ Bednar, et al., ${ }^{3}$ reported a mortality rate of $27 \%$ (nine of 33 patients) in the management of odontoid fractures in this era of "modern medicine," typically due to respiratory compromise. Lewallen, et al., ${ }^{10}$ identified respiratory arrest in four patients of whom three died as a consequence of traction for posteriorly displaced odontoid fractures. They proposed that following fracture manipulation subsequent neurological compromise causing apnea occurred. However, radiographs taken during the respiratory event in two of these patients did not demonstrate a reduction in the spinal canal diameter by the odontoid fragment; neither patient suffered a neurological decline. Therefore, an alternative mechanism for respiratory compromise should be considered.

In our series, 14 of 53 patients with displaced Type II odontoid fractures developed respiratory distress shortly after skeletal traction was applied. Thirteen of the 14 had posteriorly displaced odontoid fractures that were treated with flexion traction. Nine of these 13 patients required endotracheal intubation or tracheostomy for airway management. Four patients could not initally be intubated, and two patients died of anoxia caused by a delay in securing an airway. Although the magnitude of displacement was greater for posteriorly displaced fractures, no patient suffered neurological deterioration related to fracture displacement that may have contributed to respiratory failure. Respiratory compromise most often occurred in patients treated acutely with closed flexion reduction for a posteriorly displaced odontoid fracture.

Nearly all patients with respiratory distress sustained their injuries after falls. In contrast, many patients without respiratory compromise sustained high velocity injuries such as those that might be received in motor vehicle accidents. If the occurrence of respiratory distress was related to the extent of trauma, one might expect patients with high velocity injuries to have a higher frequency of respiratory decompensation than was observed.

Consequently, the pathogenesis of respiratory decompensation is most likely due to upper airway obstruction. 


\section{Respiratory compromise with Type II odontoid fracture}

Patients with axial injuries typically develop acute retropharyngeal edema from soft-tissue injury. This has been observed as widening of the prevertebral tissues on lateral cervical radiographs, axial computerized tomography scanning and magnetic resonance imaging. The axis and adjacent soft tissue are situated posterior to the pharynx and trachea. Axial skeletal traction with concurrent flexion causes posterior displacement of the trachea. After acute cervical trauma, particularly in the setting of retropharyngeal hematoma, subsequent flexion traction can further compress the trachea, compromising the patency of the upper airway with resultant impairment of ventilation and clearing of secretions. Acute respiratory distress may be fatal, as observed in two of the patients with posteriorly displaced Type II odontoid fractures who sustained irreversible anoxic injury because an airway could not initally be secured. Finally, many of these patients are elderly and are at even greater risk of respiratory distress due to a sixfold decrease in their protective airway reflexes secondary to aging. ${ }^{12}$

In conclusion, frequent respiratory failure was observed during the acute closed management of displaced odontoid fractures. Posteriorly displaced fractures were more commonly associated with respiratory distress. These patients were managed in flexion traction to achieve reduction. Consequently, we recommend attempts at closed reduction with neutral traction for posteriorly displaced fractures. However, if reduction requires a flexed posture, then we recommend consideration of elective fiberoptic nasotracheal intubation to ensure airway protection during spinal manipulation.

\section{Acknowledgment}

The authors would like to thank Jacqueline Sullivan, R.N., Ph.D. for her help with the statistical analysis.

\section{References}

1. Anderson LD, D'Alonzo RT: Fractures of the odontoid process of the axis. J Bone Joint Surg (Am) 56:1663-1674, 1974

2. Apuzzo MLJ, Heiden JS, Weiss MH, et al: Acute fractures of the odontoid process. An analysis of 45 cases. J Neurosurg 48: 85-91, 1978

3. Bednar DA, Parikh J, Hummel J: Management of type II odontoid process fractures in geriatric patients: a prospective study of sequential cohorts with attention to survivorship. J Spinal Disord 8:166-169, 1995

4. Clark CR, White AA III: Fractures of the dens. A multicenter study. J Bone Joint Surg (Am) 67:1340-1348, 1985

5. Dunn ME, Seljeskog EL: Experience in the management of odontoid process injuries: an analysis of 128 cases. Neurosurgery 18:306-310, 1986

6. Ekong CE, Schwartz ML, Tator $\mathrm{CH}$, et al: Odontoid fracture: management with early mobilization using the halo device. Neurosurgery 9:631-637, 1981

7. Hadley MN, Dickman CA, Browner CM, et al: Acute axis fractures: a review of 229 cases. J Neurosurg 71:642-647, 1989

8. Husby J, Sorensen KH: Fracture of the odontoid process of the axis. Acta Orthop Scand 45:182-192, 1974

9. Jefferson G: Fracture of the atlas vertebra. Br J Surg 7: 407-422, 1920

10. Lewallen RP, Morrey BF, Cabanela ME: Respiratory arrest following posteriorly displaced odontoid fractures. Case reports and review of the literature. Clin Orthop (188): 187-190, 1984

11. Lipson SJ: Fractures of the atlas associated with fractures of the odontoid process and transverse ligament ruptures. J Bone Joint Surg (Am) 18:940-943, 1977

12. Pantoppidan H, Beecher HK: Progressive loss of protective reflexes in the airway with the advance of age. JAMA 174: 77-81, 1960

13. Przybylski GJ, Welch WC: Longitudinal atlantoaxial dislocation with type III odontoid fracture. Case report and review of the literature. J Neurosurg 84:666-670, 1996

14. Schatzker J, Rorabeck CH, Waddell JP: Fractures of the dens (odontoid process). An analysis of thirty-seven cases. J Bone Joint Surg (Br) 53:392-405, 1971

Manuscript received April 26, 2000.

Accepted in final form May 24, 2000.

This work was supported in part by the Regional Spinal Cord Injury Center of Delaware Valley Model SCI System Grant No. H133 N00027 to Thomas Jefferson University Hospital from the National Institute for Disability Research and Rehabilitation.

Address reprint requests to: Gregory J. Przybylski, M.D., 1015 Chestnut Street Suite 1400, Philadelphia, Pennsylvania 19107. email: gregory.przybylski@mail.tju.edu. 